\title{
Fatigue Strength Analysis on the Automobile Stabilizer Bar Based on ANSYS
}

\author{
Peng Zhang ${ }^{*}, 1$, Bao $\mathrm{Xu}^{1}$, Shi Zhou ${ }^{1}$ and $\mathrm{Le} \mathrm{He}^{2}$ \\ ${ }^{I}$ School of Mechanical Electronic and Information Engineering, China University of Mining and Technology, Beijing, \\ Beijing, 100083, China \\ ${ }^{2}$ State Key Laboratory of Automotive Safety and Energy, Tsinghua University, Beijing, 100084, China
}

\begin{abstract}
Stabilizer bar is an important component of the vehicle's independent suspension system and plays an important role in the safety traffic. Therefore, the research on fatigue strength characteristics of the automobile stabilizer bar is very important. In this paper, the finite element model is established for the automobile stabilizer bar by utilizing ANSYS finite element analysis software. The automobile stabilizer bar's strength and stiffness are analyzed with the finite element method. It is ensured that the stabilizer bar meets the static strength requirements. At last, the fatigue simulation analysis is carried out. The simulation results illustrate that the fatigue life of the stabilizer bar is about 673400 times and that it meets the fatigue life requirements which must be at least 500000 times in the fatigue test of the stabilizer bar.
\end{abstract}

Keywords: ANSYS, fatigue strength, finite element analysis, stabilizer bar.

\section{INTRODUCTION}

Automobile stabilizer bar is an important safety component of the automobile's independent suspension system which is used to improve the operation stability of the automobile when the automobile is turning or encountering resistance. Its performance directly affects the driving safety. According to statistics, the rollover probability of the vehicle equipped with the stabilizer bar in the poor road having sharp turn conditions can be reduced by $60 \% \sim 80 \%$ [1]. It is necessary to study the static force and fatigue characteristics of the stabilizer bar as the analysis results can provide an important reference for the automotive stabilizer bar design.

Stabilizer bar is made of the spring steel having a Ushaped cross on the vehicle's front or rear suspension. Both ends of the shaft are supported on the frame through the sleeve; the shaft can be rotated within the sleeve to form a hinge support. Meanwhile, in order to prevent the occurrence of the stabilizer bar motion interference in the process of movement, this hinge support of the finite element model should be able to move longitudinally along the frame and form a living hinge bearing. The rod ends along the longitudinal direction are connected with the suspension's spring support through the ball hinge.

When the vehicle body only has vertical movement by the symmetrical loads and both sides of the suspension have equivalent deformations, the stabilizer bar rotates in

*Address correspondence to this author at the School of Mechanical Electronic and Information Engineering, China University of Mining and Technology, Ding No.11, Xueyuan Road, Haidian District, Beijing, Postcode: 100083, China; Tel: 01062331246 ;

E-mail: zhangpeng@cumtb.edu.cn the sleeve which moves along the longitudinal direction and does not have any stabilizing effect. In this way, each part of the stabilizer bar is not forced. When the two sides of the suspension's deformation are unequal, the vehicle body rolls. This causes the longitudinal parts of the stabilizer bar ends to deflect in different directions and the stabilizer bar is then subjected to torsion [2]. The structure and location of the stabilizer bar in the automotive suspension system are shown in Fig. (1).

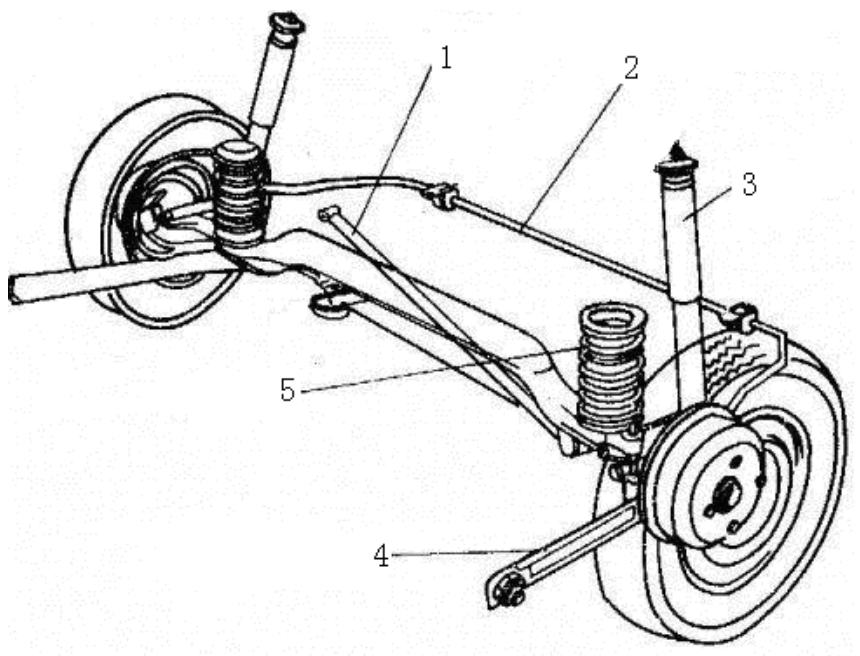

Fig. (1). Structure and location of the stabilizer bar in the automobile suspension system.

The stabilizer bar in the actual work is often subjected to different sizes of torque effect. Certain parts of the stabilizer bar result in fatigue failure with the increase in the amount of stress. Fatigue life of the stabilizer bar is an important factor that must be considered in the design therefore, conducting fatigue simulation analysis on the stabilizer bar is necessary. 
In this paper, the static force and fatigue simulation analyses on a developed stabilizer bar have been conducted by means of ANSYS software.

\section{FINITE ELEMENT MODEL OF THE STABILIZER BAR}

Simulation test was carried out at the stabilizer bar end. Travels as shown in Fig. (2) were located at the stabilizer bar end. The test simulated the deformation effect of the stabilizer bar after the vehicle was under shock load. The period loads were exerted at the stabilizer bar ends and induced vertical displacement of the two ends as $+34.7 \mathrm{~mm}$ $\&+34.7 \mathrm{~mm}$ and $-55.4 \mathrm{~mm} \&+55.4 \mathrm{~mm}$, respectively.

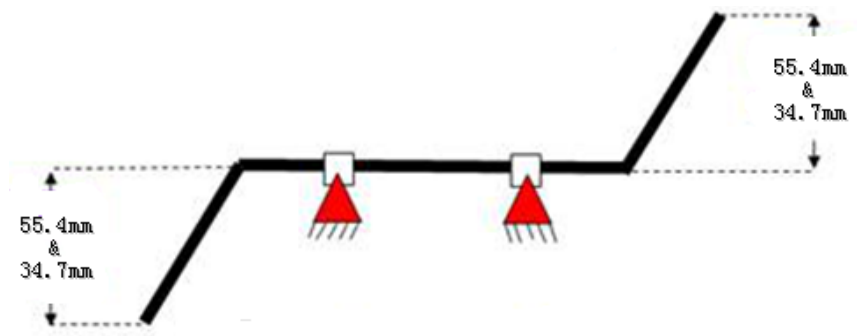

Fig. (2). Travels at the stabilizer bar end of the component test.

\subsection{Geometric Modeling}

ANSYS consists of a complete data interface and can share data with three-dimensional graphics software PRO-E. The stabilizer bar is made from the cold drawn steel pipe whose outside diameter is $\Phi 25 \mathrm{~mm}$. Depending on the geometric parameters of the stabilizer bar, firstly a threedimensional solid model is built of the stabilizer bar with PRO-E software, then ANSYS data interface is used and the geometry data files are generated by the PRO-E software into ANSYS. Finally, the entity model is transformed into the finite element model.

\subsection{Material Parameters}

The stabilizer bar's materials in the simulation are made of the spring steel $60 \mathrm{Si} 2 \mathrm{MnA}$. The density of this material is $7740 \mathrm{~kg} / \mathrm{m}^{3}$, the elastic modulus is $2.06 \times 10^{5} \mathrm{Mpa}$, and the Poisson's ratio is 0.29 [3].

\subsection{The Finite Element Model of the Stabilizer Bar}

As the structure of the vehicle's stabilizer-car was relatively simple, a solid element free meshing was used. Free meshing on the body automatically generated tetrahedral mesh. In order to obtain better accuracy, both the quadratic displacement and quadratic tetrahedral element (Solid92 unit) were used because the cross-section of the stabilizer bar possessed more turning. Tetrahedral elements consist of plasticity, creep, swelling, stress steel, large deformation and high tension. They have the strong ability to adapt to the complex models. Each unit consists of 10 nodes. Each node has three translational degrees of freedom: displacement of $\mathrm{x}, \mathrm{y}$, and $\mathrm{z}$ direction $[4,5]$. If the hexahedral elements were chosen, this method must have automatically degenerated hexahedral cells to tetrahedral elements with the same order. The linear tetrahedral units after degradation have much stronger stiffness and poorer accuracy. So it was best not to choose the linear hexahedral elements having no intermediate nodes, such as Solid 45 unit. If the quadratic hexahedral elements (such as Solid 95 unit) were chosen, the number of nodes would have been consistent with the hexahedral prototype units and only there would have been multiple nodes in the same location because it was the degenerated form. The free meshing for the finite element model of the stabilizer bar is shown in Fig. (3).

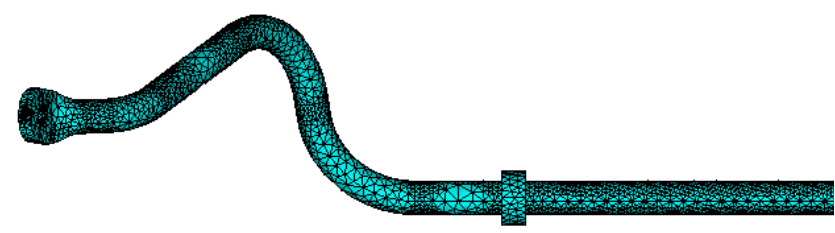

Fig. (3). Meshing for the finite element model of the stabilizer bar.

\subsection{Load and Constraint Handling}

Fig. (4) is a simplified torsion diagram of the stabilizer bar. $2 \& 3$ are the contact points between the stabilizer bar and the sleeve. In order to simplify the model, these two regions are simplified into two clamping points. The equal and opposite perpendicular forces are applied on points $1 \&$ 4 respectively. In the finite element model, points $2 \& 3$ are treated as degrees of freedom constraint; and the forces of points $1 \& 4$ are converted to the displacement loads.

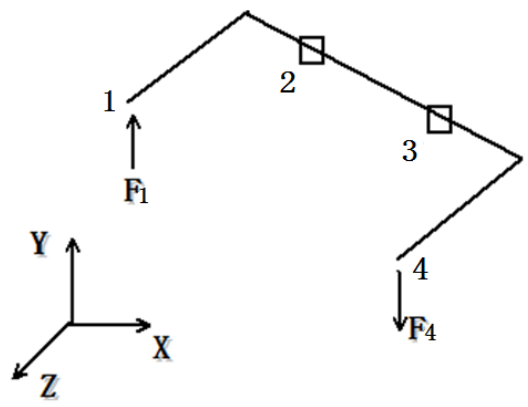

Fig. (4). Simplified torsion diagram of the stabilizer bar.

\section{STATIC ANALYSIS OF THE STABILIZER BAR}

According to the stress analysis and the actual working conditions of the stabilizer bar, the displacement constraints were imposed on the two connection surface between the stabilizer bar and the sleeve to limit the movement and rotation along $\mathrm{y}$-axis and $\mathrm{z}$-axis. The oppositely vertical displacement loads of $34.7 \mathrm{~mm}$ were applied on the two ends of the stabilizer bar. The obtained deformation comparison of the stabilizer bar after conducting ANSYS solving is shown in Fig. (5).

As can be seen from Fig. (5), deformations of both ends of the stabilizer bar were bigger. There was a little deformation on the intermediate section of the stabilizer bar because of the torsion effect. The displacement nephogram is shown in Fig. (6) obtained by the statics analysis for the stabilizer bar under the oppositely vertical displacement loads of $34.7 \mathrm{~mm}$. 
DISPLACEMENT

STEP $=1$

SUB $=1$

$\operatorname{DMX}=.04301$

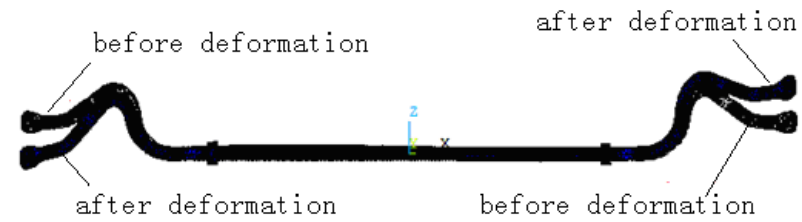

Fig. (5). Comparison of the deformation for the stabilizer bar.

$$
\begin{aligned}
& \text { NODAL SOLUTION } \\
& \text { STEP }=1 \\
& \text { SUB }=1 \\
& \text { TIME }=1 \\
& \text { USSM } \\
& \text { RSYS }=0 \quad \text { (AVG) } \\
& \text { DMX }=.035754 \\
& \text { SMX }=.035754
\end{aligned}
$$

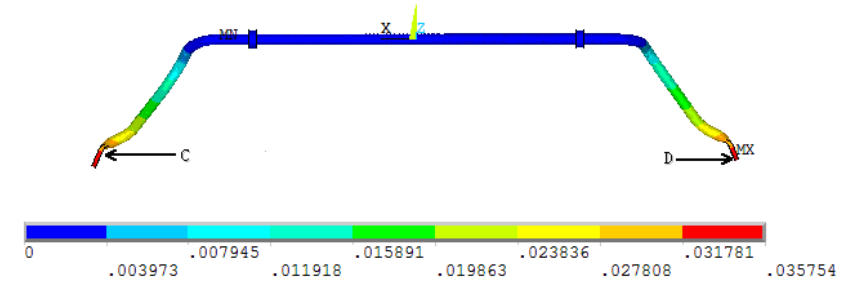

Fig. (6). Displacement nephogram of the static analysis for the stabilizer bar.

As can be seen from Fig. (6), there was a smaller deformation which was close to zero on the intermediate section of the stabilizer bar. The biggest displacement deformation was $35.8 \mathrm{~mm}$ which occurred at the ends of the stabilizer bar's longitudinal section. This was due to the fact that the vertical displacement was smaller and was close to the longitudinal axis while the vertical displacement was bigger and was away from the longitudinal axis when the roll happened. The stress nephogram is shown in Fig. (7) obtained by the statics analysis for the stabilizer bar under the oppositely vertical displacement loads of $34.7 \mathrm{~mm}$.

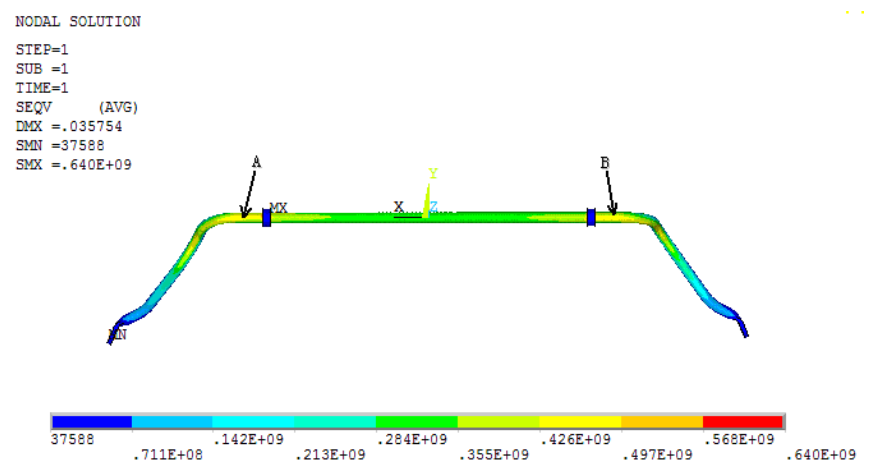

Fig. (7). Stress nephogram of the static analysis for the stabilizer bar.

As can be seen from Fig. (7), in addition to the contact points, the biggest concentrated stress was at $\mathrm{A}$ and $\mathrm{B}$ points where there was a junction between the transverse section and longitudinal section of the stabilizer bar and which involved a stress of $640 \mathrm{MPa}$. This was because the junction was constrained by the sleeve and the stabilizer-bar's crosssection encountered a turning when there was stress concentration and was also prone to fatigue failure in the actual situation.

As mentioned above, the oppositely vertical displacement loads of $54.4 \mathrm{~mm}$ were applied on the two ends of the stabilizer bar. The obtained deformation comparison of the stabilizer bar after carrying out of ANSYS solving is shown in Fig. (8).

$$
\begin{aligned}
& \text { DISPLACEMENT } \\
& \text { STEP }=1 \\
& \text { SUB }=1 \\
& \text { TIME }=1 \\
& \text { DMX }=.068553
\end{aligned}
$$

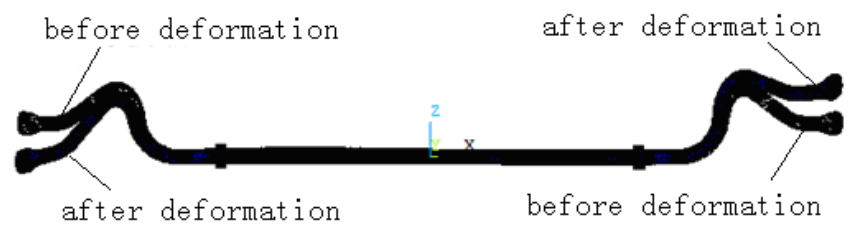

Fig. (8). Comparison of the deformation for the stabilizer bar.

As can be seen from Fig. (8), deformations of both the ends of the stabilizer bar were bigger. There was a little deformation on the intermediate section of the stabilizer bar because of the torsion effect. The displacement nephogram is shown in Fig. (9) obtained by the statics analysis for the stabilizer bar under the oppositely vertical displacement loads of $54.4 \mathrm{~mm}$.

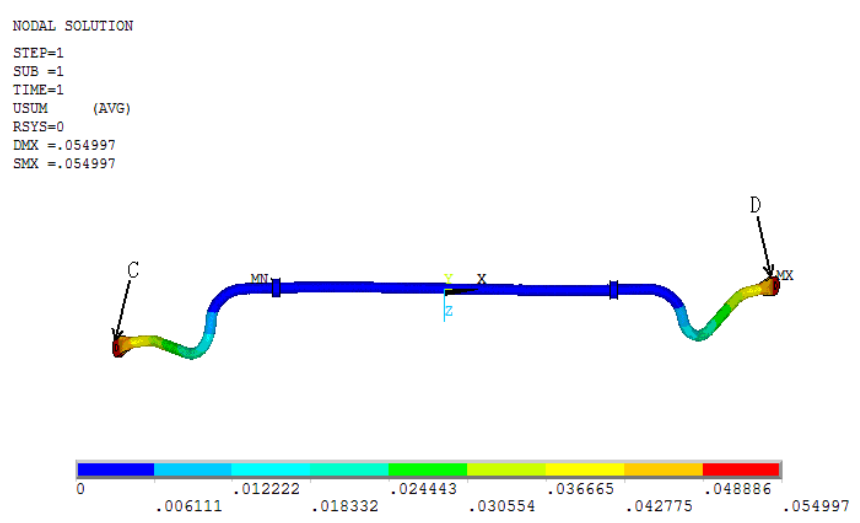

Fig. (9). Displacement nephogram of the static analysis for the stabilizer bar.

As can be seen from Fig. (9), the biggest displacement deformation was $55 \mathrm{~mm}$ which occurred at the ends of the stabilizer bar's longitudinal section. There was a smaller deformation which was close to zero on the intermediate section of the stabilizer bar. The stress nephogram is shown in Fig. (10) by the statics analysis for the stabilizer bar under the oppositely vertical displacement loads of $54.4 \mathrm{~mm}$.

As can be seen from Fig. (10), in addition to the contact points, the biggest concentrated stress was at $\mathrm{A}$ and $\mathrm{B}$ points where there was a junction between the transverse section and longitudinal section of the stabilizer bar which was a stress of $914 \mathrm{MPa}$. The stabilizer bar was mainly affected by the torsion which was equivalent to the torsion spring. Its material was $60 \mathrm{Si} 2 \mathrm{MnA}$ whose mechanical properties included the tensile strength $\sigma \mathrm{b}$ was greater than or equal to $1570 \mathrm{Mpa}$ and the yield strength $\sigma_{\mathrm{s}}$ was greater than or 


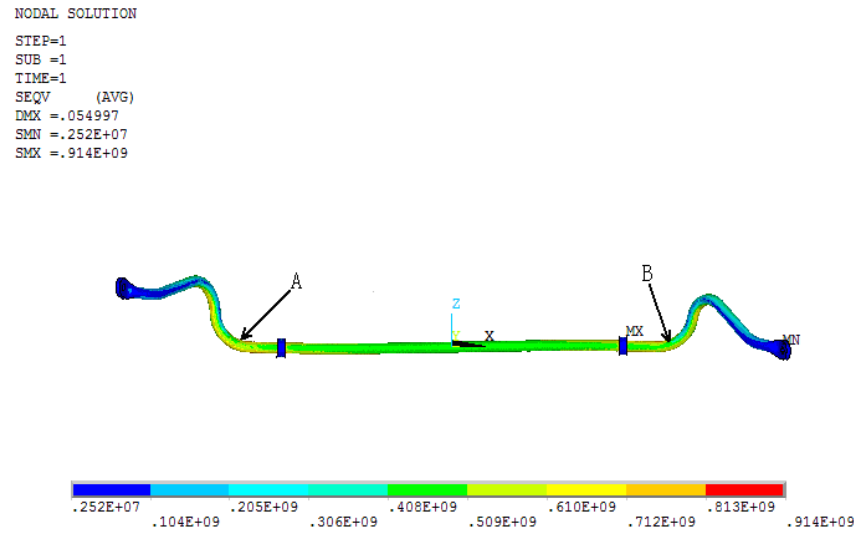

Fig. (10). Stress nephogram of the static analysis for the stabilizer bar.

equal to $1375 \mathrm{Mpa}$ [3]. The maximum stress of the stabilizer rod was within the tensile strength and yield strength. Thus, the requirements of static strength were met.

\section{FATIGUE ANALYSIS}

Fatigue can bear the disturbance stress at some point and form the crack after enough circulation disturbances or involve the development process of the local permanent structural changes occurring in the completely ruptured material. The stress or strain cycle number which the component fatigue damage needs under the cyclic loading is called the fatigue life. The fatigue performance for the formation stage of the high cycle fatigue crack is often characterized as S-N curve. Where $\mathrm{S}$ is the stress level, $\mathrm{N}$ is the fatigue life. S-N curve required evaluation throughout the test. After statistically analyzing the test results, the mapping relation curve between the stress and the safe life according to the safe life of a survival rate $\mathrm{P}$ is known as the $\mathrm{P}-\mathrm{S}-\mathrm{N}$ curve. The relational curve between the stress and the fatigue life of the survival rate $50 \%$ is called the median S-N curve which is also referred to as S-N curve. Practice showed that the fatigue life had a larger disperse so statistical analysis must be carried out to evaluate the problem of the survival rate. The fatigue life $\mathrm{Np}$ with the survival rate of $\mathrm{P}$ (such as 95\%, 99\%, 99.9\%) indicated that the fatigue life of the individuals with the survival rate of $\mathrm{P}$ in the maternal was more than $\mathrm{Np}$ while the probability of failure was equal to (1-P). S-N curve which was obtained by the conventional fatigue test included the curve of $\mathrm{P}=50 \%$. The $\mathrm{S}-\mathrm{N}$ curve of $60 \mathrm{Si}_{2} \mathrm{Mn}$ was obtained through access to the relevant information and then the corresponding P-S-N curve of the material was drawn as shown in Fig. (11).

The fatigue analysis was simulated on the basis of stress analysis by means of Fatigue Tool of ANSYS software. Fatigue Tool used the stress-life method which was the method of estimating the service life on the basis of $\mathrm{S}-\mathrm{N}$ curve and can obtain the total life directly. The fatigue analysis using ANSYS incorporated the parameter setting for the material fatigue performance, the fatigue analysis and the assessment of the fatigue results. The post-processing stress results were used to determine the fatigue life consumption coefficient of the unit or shell element model in ANSYS software. Firstly, the location of maximum called the stress

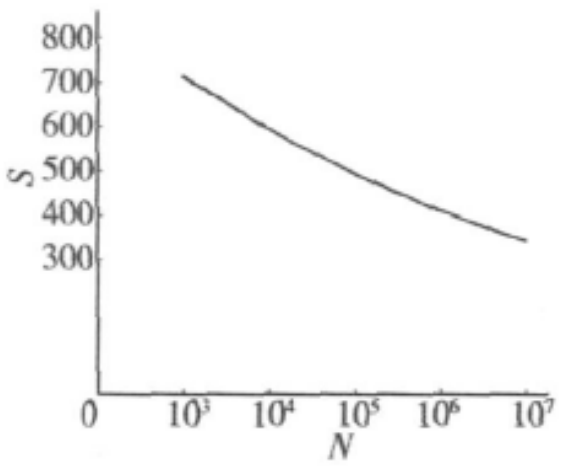

Fig. (11). P-S-N curve of $60 \mathrm{Si}_{2} \mathrm{Mn}$.

point was found and stored after the stress calculation was completed and the ends' stress concentration point was removed. The second loading solution was carried out whose direction of two displacement loads was opposite to the first loading. Then the solution results were saved and sent into POST1 after the calculation was completed. At last, the S-N curve of the material calculated by the front was inputted to the S-N table of ANSYS as shown in Fig. (12). Fatigue life of the stabilizer bar required that the cycles must be at least 500000 times in the amplitude of $54.4 \mathrm{~mm}$ and frequency of $3 \mathrm{~Hz}$ conditions. Accordingly, the corresponding parameters were inputted in the Fatigue item of ANSYS post-processing POST1. The results showed that the allowable cycles of the stabilizer bar were 6730000 times and the use coefficient was 0.36749 when the cycles were 500000 times. To meet the usage requirements, it was proposed to increase the strength of materials or optimize the structure of the stabilizer bar.

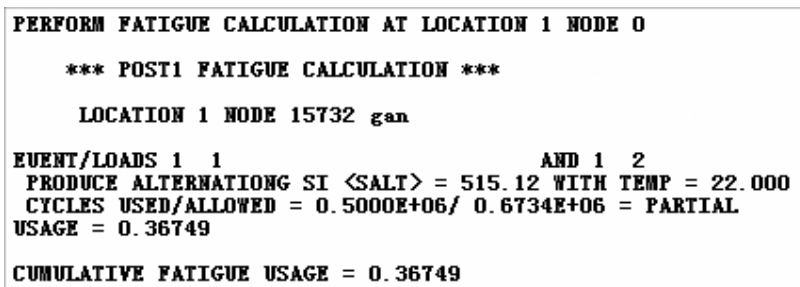

Fig. (12). Fatigue strength calculations.

\section{CONCLUSION}

The finite element model was established for the automobile stabilizer bar by utilizing ANSYS finite element analysis software in the paper. The automobile stabilizer bar's strength and stiffness were analyzed through the finite element method and the fatigue simulation analysis was carried out. The following conclusions were drawn:

(1) The design of the stabilizer bar can meet the requirements of the fatigue life.

(2) It is suggested to increase the strength of the materials or optimize the structure of the stabilizer bar for pressing. 
(3) The CAD/CAE simulation analysis software can be used to rapidly judge stress, fatigue life and reliability of the mechanical product.

(4) The design cycle of the product can be greatly shortened through this method which can enable a quicker response to the selection of the materials and the optimization of the structure and can be used for the design of other similar mechanical components.

\section{CONFLICT OF INTEREST}

The authors confirm that this article content has no conflict of interest.

\section{ACKNOWLEDGEMENTS}

This work was financially supported by the Fundamental Research Funds for the Central Universities (2009QJ01) and University Students' Innovative Training Program.

\section{REFERENCES}

[1] J. Y. Xiang, "The manufacturing of automotive stabilizer bar", Metal World, vol. 2, pp. 32-34, 2008.

[2] S. G. Lie, "Influence of stabilizer on the dynamic and static response in bus body", Machinery Manufacturing Engineer, vol.2, pp.123-125, 2005.

[3] Engineering materials practical manual $\left(2^{\text {nd }}\right.$ ed. $)$, Beijing: Strandards Press of China, 2001, pp. 356-363.

[4] L. S. Hong, and L. Fang. "Fatigue reliability analysis on automotive stabilizer bar", Machinery Design \& Manufacture, vol.1, pp.41-43, 2012.

[5] H. Kang, and Y. D. Rong, "Fatigue analysis on the automobile transverse stability rod based on ANSYS", Journal of Machine Design, vol. 12, pp. 66-68, 2008. 